\title{
Avaliação da qualidade de vida e síndrome de burnout em professores universitários
}

\author{
Evaluation of the quality of life and burnout syndrome in university professors
}

\author{
Isabela Maria Melo Miranda ${ }^{1}$ \\ Orcid: https://orcid.org/0000-0002-9878-8996
}

Heloísa Rodrigues Soares da Silva ${ }^{3}$

Orcid: https://orcid.org/0000-0001-6429-5424

Raquel de Oliveira Santos 5

Orcid: https://orcid.org/0000-0002-1466-7338
Helen Hana Fernandes Tavares ${ }^{2}$

Orcid: https://orcid.org/0000-0003-1749-5508

\author{
Monise Santana Braga ${ }^{4}$ \\ Orcid: https://orcid.org/0000-0002-6444-1521
}

Renato Canevari Dutra da Silva ${ }^{6}$

Orcid: https://orcid.org/0000-0002-6428-2823

Heloísa Silva Guerra ${ }^{7}$

Orcid: https://orcid.org/0000-0002-0617-8112

\begin{abstract}
Introdução: A expressão qualidade de vida envolve parâmetros objetivos e subjetivos, como a saúde e outros fatores, sendo sua compreensão algo individual e dinâmico. Os professores, por sua dinâmica de trabalho, estão propensos a desenvolverem a Síndrome de Burnout e terem sua qualidade de vida afetada negativamente Métodos: Estudo transversal com docentes do ensino superior, mediante avaliação das características sociodemográficas, comportamentais, qualidade de vida pelo WHOQOL-bref e presença de Burnout pelo Maslach Burnout Inventory - Educators Survey. Além da análise descritiva, foram utilizados o coeficiente de correlação intraclasse (CCI), o alfa de Cronbach e o coeficiente de correlação de Pearson. Resultados: Os resultados dos 22 participantes apontaram maioria de docentes do sexo masculino, idade média de 36,4 anos, casados e que trabalham como docente, em média, 29,5 horas por semana. Identificou-se uma correlação negativa entre exaustão e os domínios físico $(p=0,005)$, psicológico $(\mathrm{p}=0,015)$ e geral $(\mathrm{p}=0,009)$, sugerindo redução da qualidade de vida; e uma correlação positiva entre a eficácia profissional e domínio psicológico $(\mathrm{p}=0,023)$, sugestivo de que a percepção positiva de eficácia profissional eleva a QV entre os professores. Conclusão: A prevalência da síndrome de Burnout entre os docentes universitários foi baixa, embora tenham sido identificados fatores que interferem na $\mathrm{QV}$, tanto positiva quanto negativamente. Os achados podem auxiliar na compreensão das características desse público e na elaboração de estratégias preventivas dessas condições.
\end{abstract}

Palavras-chave: qualidade de vida; docentes; saúde mental.

\footnotetext{
Abstract

${ }^{1}$ Universidade de Rio Verde. E-mail: isabela.mmm@hotmail.com

${ }^{2}$ Universidade de Rio Verde. E-mail: helenhana.ft@gmail.com

${ }^{3}$ Universidade de Rio Verde. E-mail: heloisa300494@gmail.com

${ }^{4}$ Universidade de Rio Verde. E-mail: monise.santana@gmail.com

${ }^{5}$ Universidade de Rio Verde. E-mail: osantosraquel@gmail.com

${ }^{6}$ Universidade de Rio Verde. E-mail: renatocanevari@unirv.edu.br

${ }^{7}$ Universidade de Rio Verde. E-mail: heloisasguerra@gmail.com
}

Introduction: The expression quality of life involves objective and subjective parameters, such as health and other factors, and its understanding is somewhat individual and dynamic. Teachers, due to their work dynamics, are prone to develop Burnout Syndrome and have their 
quality of life negatively affected. Objective: To evaluate the presence of Burnout Syndrome among professors of an undergraduate medical course and its correlation with quality of life. Methods: Cross-sectional study with higher education teachers, by assessing sociodemographic and behavioral characteristics, quality of life by the WHOQOL-bref and the presence of Burnout by the Maslach Burnout Inventory - Educators Survey. In addition to the descriptive analysis, the intraclass correlation coefficient (ICC), Cronbach's alpha and Pearson's correlation coefficient were used. Results: The results of the 22 participants showed a majority of male teachers, with an average age of 36.4 years, married and working as a teacher, on average, 29.5 hours per week. A negative correlation was identified between exhaustion and the physical $(p=0.005)$, psychological $(p=0.015)$ and general $(p=0.009)$ domains, suggesting a reduction in quality of life; and a positive correlation between professional effectiveness and psychological domain $(\mathrm{p}=0.023)$, suggesting that the positive perception of professional effectiveness raises QOL among teachers. Conclusion: The prevalence of Burnout syndrome among university professors was low, although factors that interfere in QOL, both positive and negative, have been identified. The findings can assist in understanding the characteristics of this audience and in the development of preventive strategies for these conditions.

Keywords: quality of life; faculty; mental health.

\section{Introdução}

A Organização Mundial de Saúde (OMS) define qualidade de vida (QV) como a "percepção do indivíduo de sua posição na vida no contexto de sua cultura e sistema de valores em que vive e em relação aos seus objetivos, expectativas, padrões, preocupações e desejos".

$\mathrm{O}$ conceito de qualidade de vida começou a ser utilizado no contexto pós Segunda Guerra Mundial, nos Estados Unidos da América, com o único objetivo de descrever os bens materiais adquiridos, tais como viagens e investimentos. Com o tempo esse conceito foi se ampliando e incorporando alguns indicadores para sua mensuração, como o produto interno bruto e a renda per capita. Posteriormente, passou a incorporar também o conceito social e a mensurar o desenvolvimento por meio de outros indicadores, tais como moradia, saúde e educação ${ }^{2}$.

Atualmente, a expressão qualidade de vida envolve parâmetros objetivos e subjetivos, como saúde, urbanismo, lazer, esportes, educação, meio ambiente, segurança, entretenimento, novas tecnologias e tudo o que se relacione com o ser humano, sua cultura e seu meio. A compreensão da qualidade de vida é individual e dinâmica ${ }^{3}$.

Os professores estão expostos a diversos fatores prejudiciais para sua qualidade de vida, sendo eles a violência, falta de segurança, salas de aula superlotadas, inexistência de autonomia, sentimento de frustração, salários inadequados, carga horária excessiva, estudantes mal preparados que, por si só, apresentam risco de incapacidade de gerir o estresse $^{3,4,5}$.

Esse excesso de estresse pode causar inúmeros fatores como insônia, fadiga, irritabilidade, ansiedade e até depressão ${ }^{6}$. Além disso, relaciona-se com baixo desempenho no trabalho interferindo na relação professor-aluno, gerando questionamento sobre a escolha profissional e podendo desencadear a Síndrome de Burnout $(\mathrm{SB})^{5,7}$. Esse termo foi aperfeiçoado por Maslach e Jackson, sendo definido como um transtorno mental composto por exaustão emocional, despersonalização e baixa realização pessoal, causada pelo prolongamento de situações de desgaste relacionado ao trabalho ${ }^{8}$.

Embora possa acometer profissionais de diferentes categorias, a SB tem ocorrido de forma mais acentuada entre profissionais da área da saúde, segurança e educação. Ao longo dos anos algumas pesquisas têm demonstrado a presença da SB entre professores, o que desperta maior preocupação para com esse público ${ }^{7,9-14}$.

A Organização Internacional do Trabalho (OIT), considera a profissão 
docente como uma das mais estressantes, e com forte presença de elementos que podem predispor à $\mathrm{SB}^{5}$. Uma metanálise apontou que a insatisfação com o trabalho apareceu associada a problemas mentais e psicológicos, relacionando-se em maior grau com o Burnout, depressão, ansiedade e autoestima $^{14}$.

Um levantamento realizado em 2017, sobre os principais resultados de pesquisas realizadas no tocante aos fatores relacionados à SB em professores no Brasil, apontou que a exaustão emocional aumenta ao acreditar que a profissão está interferindo na vida pessoal, considerar a profissão menos interessante do que quando iniciou, trabalhar com muitos alunos, além da carga horária exercida. Já a despersonalização aumenta com o mau comportamento dos alunos e com o maior tempo de serviço. A realização profissional diminui conforme aumenta o tempo de exercício profissional, maior carga horária e o número de alunos ${ }^{15}$.

Estudo transversal realizado no Rio de Janeiro com professores universitários demonstrou que um quarto dos participantes apresentavam sintomas compatíveis com a $\mathrm{SB}$, sendo a desumanização um item reportado por $30,6 \%$ dos docentes ${ }^{13}$. Em Belém, estudo realizado com professores médicos identificou a SB e um alto índice de despersonalização em mais de $50 \%$ dos entrevistados ${ }^{9}$, e em outra pesquisa com professores universitários paulistas 11,2\% dos participantes apresentaram sintomas moderados da $\mathrm{SB}$ e $3 \%$ sintomas graves, com destaque para as dimensões decepção no trabalho e exaustão emocional ${ }^{10}$.

A Saúde do Trabalhador é um direito fundamental, protegido pela Constituição Federal brasileira, a qual atribui ao Sistema Único de Saúde (SUS) o dever de executar ações que visem garanti-lo. Nesse contexto, diversas normativas regulamentam o tema, sendo a notificação compulsória de casos que envolvam acidentes de trabalho e doenças profissionais, uma das mais importantes para as políticas públicas de saúde. A SB faz parte da lista de transtornos mentais e comportamentais relacionados ao trabalho, que são de notificação compulsória de acordo com o Ministério da Saúde $^{16}$. Notificar os casos é extremamente relevante na medida em que possibilita a instauração de um processo de informaçãodecisão-ação quanto a medidas de prevenção e controle das doenças ou agravos à saúde.

Ademais, a realização de estudos sobre essa temática pode colaborar para $o$ conhecimento acerca do perfil dos docentes universitários brasileiros e auxiliar na compreensão dos fatores que podem interferir em sua saúde e bem-estar. O objetivo desse estudo foi avaliar a presença da Síndrome de Burnout entre os docentes de um curso de graduação em Medicina e sua correlação com a qualidade de vida.

\section{Materiais e Métodos}

Estudo transversal, com professores do curso de Medicina da Universidade de Rio Verde campus Aparecida de Goiânia realizado entre 2017 e 2018 . Identificaramse 54 docentes em atividade na faculdade de Medicina. Foram excluídos 09 professores que exerciam apenas atividades administrativas e os que estavam em período de licença (maternidade, férias e médica). Todos os demais foram convidados a participarem da pesquisa mediante abordagem presencial. Houve sete recusas e 16 docentes não entregaram o questionário respondido no prazo estipulado, mesmo após três tentativas de contato, totalizando uma amostra de 22 professores.

Para coleta de dados foram utilizados três instrumentos: um sociodemográfico e de hábitos de vida, o World Health Organization Questionnaire for Quality of Life - Brief Form (WHOQOL-bref) e o Maslach Burnout Inventory - Educators Survey (MBI-ES).

O World Health Organization Questionnaire for Quality of Life - Brief Form (WHOQOL-bref), é instrumento desenvolvido pela OMS e validado no Brasil $^{1,10}$. Trata-se de uma versão abreviada do World Health Organization 
Questionnaire for Quality of Life - 100 (WHOQOL-100), sendo constituído por 24 perguntas representantes de cada uma das 24 partes que compõem o instrumento original, divididas em quatro domínios que tem por objetivo verificar a capacidade física (sete questões), o bem-estar psicológico (seis questões), as relações sociais (três questões) e o meio ambiente onde o indivíduo está inserido (oito questões), e, ainda, duas questões gerais de QV. A versão em português do WHOQOLbref foi considerada com boa consistência interna, satisfatória em relação à validade discriminante, concorrente e de conteúdo; e teste-reteste fidedigno ${ }^{17}$.

O Maslach Burnout Inventory Educators Survey (MBI-ES), que avalia a Síndrome de Burnout, verifica como o trabalhador vivencia seu trabalho, de acordo com as três dimensões que constituem a síndrome: Exaustão Emocional (EE), Despersonalização (DP) e Realização Profissional (RP). Constitui-se de 22 itens, medidos por uma escala do tipo Likert, variando de 0 a 6 , sendo 0 (nunca), 1 (algumas vezes ao ano), 2 (uma vez ao mês ou menos), 3 (algumas vezes durante o mês), 4 (uma vez por semana), 5 (algumas vezes durante a semana) e 6 (todos os dias). Para o cálculo da variável é construída a média das pontuações obtidas em cada dimensão, o que dará o índice alcançado em cada uma delas. Altos escores de exaustão emocional e despersonalização e baixo escore em realização profissional (essa subescala é inversa) indicam alto nível de Burnout. Índices até 2,5 foram considerados como ausência de comprometimento, aqueles entre 2,6 e 3,0 comprometimento em nível moderado, e acima de 4,0 nível alto. Apesar de não haver consenso na literatura para o diagnóstico de síndrome de Burnout, utilizou-se como definição a presença de alto nível em pelo menos uma das três dimensões ${ }^{18}$. A versão brasileira do MBI apresenta os requisitos necessários em termos de consistência interna e validade fatorial para ser utilizada na avaliação da
Síndrome de Burnout em professores em nossa realidade ${ }^{19}$.

Os dados foram analisados no programa STATA, versão 14.0 (StataCorp, 2015). Realizou-se o teste de KolmogorovSmirnov com correção de Lilliefors para verificação da normalidade das variáveis quantitativas do estudo. As variáveis qualitativas foram trabalhadas descritivamente como frequência absoluta (n) e relativa (\%) e as quantitativas como média e desvio-padrão (DP), mínimo e máximo. Para as dimensões do instrumento de qualidade de vida e síndrome de Burnout, foram apresentados, ainda, mediana, IC $95 \%$ da média e intervalo interquartil (IIQ).

Para a análise da confiabilidade e consistência interna do WHOQOL-bref foram utilizados o coeficiente de correlação intraclasse (CCI) e o coeficiente de alfa de Cronbach. Para verificar a correlação entre os domínios da qualidade de vida e as dimensões do instrumento de Burnout foi utilizado o coeficiente de correlação de Pearson. Em todas as análises, valores de $p$ $<0,05$ foram considerados estatisticamente significantes.

O estudo foi aprovado pelo Comitê de Ética em Pesquisa da Fundação do Ensino Superior de Rio Verde - FESURV Universidade de Rio Verde por meio do Parecer $\mathrm{n}^{\mathrm{o}}$ 2.288.371, CAAE 69111317.0.0000.5077 em 21 de setembro de 2017.

\section{Resultados}

Neste estudo, a amostra foi composta por 22 professores universitários do curso de medicina de uma instituição pública de ensino superior, sendo seis com formações variadas no âmbito da saúde e 16 médicos. Esse número correspondeu à $40,7 \%$ dos docentes com vínculo empregatício na instituição no período avaliado.

$\mathrm{Na}$ amostra analisada, a maioria dos participantes era do sexo masculino (54,5\%), com idade média de 36,4 anos (DP $\pm 4,4)$ anos, casados $(63,6 \%)$, com tempo de 
experiência como docente em média de 39,3 meses ( $\mathrm{DP} \pm 30,3)$ e média de horas de trabalho semanal como docente de 29,5 horas (DP $\pm 12,4)$. Cerca de $63,6 \%$ dos docentes não trabalhavam em outra instituição de ensino e $81,2 \%$ exerciam outra função concomitante à de professor. Dentre os que atuavam em outras funções, $54,5 \%$ eram médicos da parte clínica, $13,6 \%$ na cirúrgica e os demais em serviços administrativos.

Cerca de 63,6\% dos participantes relataram dormir bem quase sempre e um total de $86,4 \%$ quase sempre se sentiam capazes de gerir o estresse. No tocante aos hábitos de vida, 68,2\% consumiam o recomendado de frutas/verduras, $72,7 \%$ não consumiam alimentos hipercalóricos, $86,4 \%$ realizavam exercícios físicos regulares, $86,4 \%$ nunca haviam fumado; $63,6 \%$ faziam uso regular de álcool; e $40,9 \%$ relataram possuir alguma patologia.

A Tabela 1 apresenta a análise descritiva dos domínios de QV dos professores, assim como testes de consistência interna. Verificou-se que o maior escore de QV foi no domínio ambiental $(74,1)$ e o menor no domínio social $(68,6)$.

Tabela 1. Análise dos domínios da qualidade de vida pelo WHOQOL-bref em professores universitários. Aparecida de Goiânia (GO), 2018.

\begin{tabular}{|c|c|c|c|c|c|c|c|c|}
\hline \multirow[t]{2}{*}{ Domínios } & \multirow[t]{2}{*}{ Média (DP) } & \multirow[t]{2}{*}{ IC 95\% } & \multirow[t]{2}{*}{ Mediana } & \multirow[t]{2}{*}{ IIQ } & Valor & \multirow{2}{*}{$\begin{array}{c}\text { Alfa de } \\
\text { Cronbach }\end{array}$} & \multirow[t]{2}{*}{ CCI } & \multirow[t]{2}{*}{ p-valor* } \\
\hline & & & & & Min. Máx. & & & \\
\hline Físico & $73,7(9,2)$ & $69,6-77,8$ & 75,0 & $67,8-79,5$ & $53,6-85,7$ & 0,499 & 0,419 & $<0,001$ \\
\hline Psicológico & $72,7(11,0)$ & $67,8-77,6$ & 75,0 & $65,0-80,0$ & $50,0-95,0$ & 0,530 & 0,514 & 0,011 \\
\hline Social & $68,6(17,8)$ & $60,7-76,4$ & 66,7 & $56,2-83,3$ & $33,3-100,0$ & 0,786 & 0,768 & $<0,001$ \\
\hline Ambiental & $74,1(9,7)$ & $69,8-78,4$ & 70,3 & $65,6-82,0$ & $62,5-96,9$ & 0,659 & 0,652 & $<0,001$ \\
\hline Geral & $69,3(19,6)$ & $60,6-78,0$ & 75,0 & $62,5-75,0$ & $12,5-100,0$ & 0,855 & 0,848 & $<0,001$ \\
\hline
\end{tabular}

Abreviações: DP: Desvio-padrão; IC 95\%: Intervalo de confiança de 95\%; DP: Desvio-padrão; IIQ: Intervalo interquartil; Min: Mínimo; Máx: Máximo; CCI: coeficiente de correlação intraclasse; *Teste F

A Tabela 2 apresenta a análise das dimensões da escala de Burnout dos professores do curso de medicina. Observou-se que a média dos escores para as dimensões exaustão emocional, despersonalização e realização profissional foram de 1,$9 ; 0,8$ e 4,5 , respectivamente. Apenas um professor apresentou alteração na dimensão $\mathrm{EE}(4,5 \%)$, caracterizando a presença da síndrome de Burnout.

Tabela 2. Análise dos domínios da escala de Burnout em professores universitários. Aparecida de Goiânia (GO), 2018.

\begin{tabular}{|c|c|c|c|c|c|c|c|c|}
\hline \multirow[t]{2}{*}{ Domínios } & \multirow[t]{2}{*}{ Média (DP) } & \multirow[t]{2}{*}{ IC 95\% } & \multirow[t]{2}{*}{ Mediana } & \multirow[t]{2}{*}{ IIQ } & Valor & \multirow{2}{*}{$\begin{array}{c}\text { Alfa de } \\
\text { Cronbach }\end{array}$} & \multirow[t]{2}{*}{ CCI } & \multirow[t]{2}{*}{ p-valor* } \\
\hline & & & & & Min. Máx. & & & \\
\hline Exaustão & $1,9(1,1)$ & $1,4-2,4$ & 1,8 & $1,0-2,8$ & $0-4,3$ & 0,895 & 0,890 & $<0,001$ \\
\hline Despersonalização & $0,8(0,9)$ & $0,3-1,2$ & 0,5 & $0,0-1,4$ & $0-3,6$ & 0,763 & 0,663 & $<0,001$ \\
\hline Realização profissional & $4,5(0,7)$ & $4,2-2,8$ & 4,5 & $4,0-4,9$ & $3,2-5,6$ & 0,643 & 0,485 & $<0,001$ \\
\hline
\end{tabular}

Abreviações: DP: Desvio-padrão; IC 95\%: Intervalo de confiança de 95\%; DP: Desvio-padrão; IIQ: Intervalo interquartil; Min: Mínimo; Máx: Máximo; CCI: coeficiente de correlação intraclasse; *Teste F.

A tabela 3 apresenta a correlação entre os domínios de QV e fatores da escala de Burnout. A análise dos dados indicou uma correlação negativa moderada entre exaustão e os domínios físico $(\mathrm{p}=0,005)$, psicológico $(p=0,015)$ e geral $(p=0,009)$, 
sugerindo redução da QV quanto maior a exaustão apresentada. Observou-se ainda uma correlação positiva moderada entre a realização profissional e domínio psicológico da QV ( $\mathrm{p}=0,023)$, sugestivo de que a percepção positiva de realização profissional eleva a QV entre os professores.

Tabela 3. Correlação entre os domínios de QV e fatores da escala de Burnout em professores universitários. Aparecida de Goiânia (GO), 2018.

\begin{tabular}{lccc}
\hline Variáveis & Exaustão emocional & Despersonalização & Realização Profissional \\
\hline Físico $(\mathrm{r})^{*}$ & $-0,575$ & $-0,305$ & $-0,003$ \\
p-valor & $\mathbf{0 , 0 0 5}$ & 0,167 & 0,990 \\
Psicológico $(\mathrm{r})^{*}$ & $-0,511$ & $-0,058$ & 0,483 \\
p-valor & $\mathbf{0 , 0 1 5}$ & 0,078 & $\mathbf{0 , 0 2 3}$ \\
Social $(\mathrm{r})^{*}$ & $-0,211$ & $-0,146$ & 0,120 \\
p-valor & 0,347 & 0,518 & 0,595 \\
Ambiental $(\mathrm{r})^{*}$ & $-0,257$ & $-0,215$ & 0,243 \\
p-valor & 0,248 & 0,338 & 0,277 \\
Geral $(\mathrm{r})^{*}$ & $-0,544$ & $-0,308$ & 0,234 \\
p-valor & $\mathbf{0 , 0 0 9}$ & 0,163 & 0,294 \\
\hline
\end{tabular}

*Coeficiente de correlação de Pearson.

\section{Discussão}

O perfil dos docentes do presente estudo corrobora com outros estudos da literatura focados nesse público: sexo masculino, casados, idade entre 30 e 40 anos e carga horária em torno de 30 horas semanais $^{20,21}$.

No tocante aos hábitos de vida, foi possível perceber que os docentes do presente estudo têm certa preocupação com a saúde, primando por uma alimentação de mais qualidade, prática de exercício físico e o não consumo de tabaco. Hábitos de vida saudáveis podem reduzir o risco de desenvolvimento de doenças crônicas não transmissíveis, além de contribuir positivamente em aspectos da saúde mental ${ }^{22}$.

Embora tenha demonstrado que quase sempre dormem bem, a média de horas de sono nesse grupo foi de 5,8 horas, tempo abaixo do recomendado para indivíduos adultos, que seria de 7-9 horas por dia ${ }^{23}$. Um dos motivos que pode colaborar para problemas do sono é a própria dinâmica da vida docente, marcada por inúmeras atividades como preparação e ministração de aulas, acompanhamento de alunos, elaboração de provas e atividades extras, correção de provas e o envolvimento em atividades extraclasse como pesquisa e extensão ${ }^{24}$. Os docentes, muitas vezes, sacrificam suas horas de descanso para poderem cumprir com todas as obrigações.

Os dados acerca da qualidade de vida evidenciaram que os professores do presente estudo apresentaram QV satisfatória, com melhor desempenho no domínio ambiental e pior no domínio social. Esses resultados divergiram de estudo realizado em Minas Gerais com docentes de cursos da área da saúde, em que o domínio social apresentou maior valor, sendo, portanto, o domínio menos afetado na QV dos participantes $^{3}$ e de outro estudo realizado com 203 professores do Rio Grande do Sul, onde o domínio físico foi o que apresentou maior média de escore e o ambiental a menor ${ }^{25}$.

O domínio ambiental refere-se a questões de sensação de segurança física e proteção, ambiente do lar, recursos financeiros, cuidados de saúde, participação e oportunidades de recreação e lazer, ao ambiente

físico (poluição/ruído/trânsito/clima) e transporte. Uma boa avaliação nesse item pode ser reflexo da localização do município onde o estudo foi desenvolvido pois a região metropolitana de Goiânia, na qual Aparecida de Goiânia faz parte, figura entre as melhores regiões metropolitanas do país com maior qualidade de vida ${ }^{26}$. 
Já, o domínio social diz respeito às relações interpessoais e redes de apoio social. A amostra deste estudo era predominantemente formada por profissionais médicos, e muitos deles exerciam a função assistencial concomitante ao ensino, atuando em uma dupla jornada. O tempo é um dos principais fatores que interferem na $\mathrm{QV}$ do docente, sendo que quanto maior a dedicação ao trabalho, menor é a disponibilidade para outras atividades pessoais, como usufruir de momentos com a família, amigos, atividades domésticas, cuidados com a saúde e o lazer, impactando negativamente o contexto social ${ }^{3}$.

No tocante à escala de Burnout, os resultados do presente estudo foram semelhantes a um estudo realizado com docentes de uma universidade privada do estado de São Paulo, em que exaustão emocional obteve média de escore de 1,80; despersonalização 0,84 e eficácia profissional 1,095. Em contrapartida, os achados divergiram de estudo realizado em Santa Maria, em que os 60 docentes universitários apresentaram médias dos escores de cada dimensão, sugerindo grau grave no domínio despersonalização $(4,1)$ e moderado em exaustão emocional $(2,7)$ e realização profissional $(2,5)^{27}$.

No presente estudo apenas um docente apresentou pontuação coerente com a definição de SB na dimensão $\mathrm{EE}$, sugerindo desgaste emocional e exaustão física pelo trabalho em nível grave. De acordo com um dos criadores do instrumento, essa dimensão é precursora no processo de desenvolvimento da síndrome ${ }^{28}$. Outros estudos conduzidos, utilizando-se a mesma escala para avaliação do Burnout, mas considerando a pontuação total do instrumento, um realizado no Rio Grande do Norte e o outro na Colômbia, apontaram níveis de comprometimento em cada dimensão variando de baixo a moderado entre os docentes ${ }^{29,30}$.

As variações de comprometimento entre docentes podem ocorrer e são influenciadas por muitos fatores como, por exemplo, carga horária de trabalho, experiência profissional, quantidade de cursos que leciona e número de alunos atendidos ${ }^{5,31}$.

Observou-se uma correlação negativa entre exaustão e os domínios físico, psicológico e geral, sugerindo que quanto maior a exaustão menor a qualidade de vida dos docentes do presente estudo. O domínio físico envolve a percepção do docente frente a dor e desconforto, sono, repouso, mobilidade, atividades cotidianas, dependência de medicação e outros tratamentos e capacidade para o trabalho. $\mathrm{O}$ domínio psicológico refere-se à questão de sentimentos positivos, pensamento, aprendizado, memória, concentração, autoestima, imagem corporal e aparência, sentimentos negativos e espiritualidade. De fato, a exaustão, que se caracteriza por sentimentos de sobrecarga e esgotamento de recursos físicos e emocionais, pode se refletir em dor física e afetar as relações interpessoais e a qualidade de vida do docente em vários aspectos ${ }^{2,20}$.

A exaustão pode ainda desencadear sentimento de apatia, desânimo e impulsionar dificuldades de visualizar estratégias para recobrar motivação, lazer e saúde, comprometendo a relação com alunos e até o compartilhamento do conhecimento $^{32}$.

Um outro ponto que se mostrou relevante no presente estudo diz respeito à correlação positiva entre a dimensão realização profissional e o domínio psicológico. O aumento da realização profissional está associado a maior coping ativo, suporte emocional e reinterpretação positiva $^{15}$.

Alguns fatores podem se associar à realização profissional, entre eles gostar da profissão, a possibilidade de atuar em diferentes frentes como pesquisa conjugada a trabalhos de extensão com a comunidade, o reconhecimento profissional por parte dos discentes e da sociedade, a gratificação em contribuir com a formação de profissionais, a autonomia, a flexibilidade e a possibilidade de aprendizado ${ }^{33}$. 
Algumas limitações do estudo em questão devem ser consideradas quanto aos resultados, como o fato de ter delineamento transversal, número pequeno de participantes e se referir a uma instituição de nível superior apenas.

Embora a prevalência da síndrome de Burnout tenha sido baixa, é crescente a preocupação com essa classe profissional quanto ao risco de desenvolver o esgotamento profissional. Os estressores presentes no ambiente de trabalho docente são vários como salas de aula com muitos alunos, desvalorização profissional, condições inadequadas de trabalho, excesso de demandas, carga horária excessiva e falta de participação nas decisões institucionais $^{31,34,35}$.

\section{Conclusão}

Apenas um docente apresentou comprometimento compatível com a síndrome de Burnout neste estudo, indicando uma baixa prevalência na amostra estudada. Os achados apontaram fatores que interferem na QV dos docentes universitários, como maiores escores no domínio exaustão impactando negativamente e, maior percepção da realização profissional, influenciando positivamente. Pesquisas futuras acerca desse tema são importantes pois podem contribuir para melhor compreensão dos aspectos que levam ao esgotamento profissional e diminuem a QV de professores, apoiando estratégias futuras de prevenção dessas condições nesse público.

\section{Referências}

1. WHOQOL Group. Development of the World Health Organization WHOQOL-BREF quality of life assessment. The WHOQOL Group. Psychol Med. 1998; 28(3):551-8.

2. Alves PC, Oliveira AF, Paro HBMS. Quality of life and burnout among faculty members: How much does the field of knowledge matter? PLoS ONE. 2019; 14(3): e0214217.

3. Souto LES, Souza SM, Lima CA, Lacerda MKS, Vieira MA, Costa FM, Caldeira AP. Fatores Associados à Qualidade de Vida de Docentes da Área da Saúde. Rev. bras. educ. med. 2016; 40(3):452-60.

4. Ribeiro LCC, Barbosa ACR, Soares AS. Avaliação da prevalência de Burnout entre professores e a sua relação com variáveis sociodemográficas. R. Enferm. Cent. O. Min. 2015; 5(3):1741-51.

5. Prado RL, Bastianini ME, Cavalleri MZ, Ribeiro SFR, Pizi ECG, Marsicano JA. Avaliação da síndrome de Burnout em professores universitários. Revista da ABENO. 2017; 17(3):21-9.

6. Moss M, Good VS, Gozal D, Kleinpell R, Sessler CN. An official critical care societies collaborative statement: burnout syndrome in critical care health care professionals: a call for action. Crit Care Med. 2016; 44(7):1414-21.

7. Baptista MN, Soares TFP, Raad AJ, Santos LM. Burnout, estresse, depressão e suporte laboral em professores universitários. Rev. Psicol., Organ. Trab. 2019;19(1): 564-70.

8. Maslach C, Jackson SE, Leiter MP. MBI Maslach Burnout Inventory. CPP, Incorporated, 1996.

9. Gonçalves TB, Leitão AKR, Botelho BS, Marques RACC, Hosoume VSN, Neder PRB. Prevalência de síndrome de burnout em professores médicos de uma universidade pública em Belém do Pará. Rev Bras Med Trabalho. 2011;9(2):85-9.

10. Costa LST, Gil-Monte PR, Possobona RF, Ambrosano GMB. Prevalência da síndrome de Burnout em uma amostra de professores universitários brasileiros. Psicol Reflexão Crit. 2013;26(4):636-42. 
11. Dallacosta FM. Avaliação do nível de satisfação no trabalho e dos sintomas de Burnout em docentes da área da saúde [Tese] Rio Grande do Sul: Programa de Pós-Graduação da Faculdade de Medicina da Pontifícia Universidade Católica. 2014.

12. Borges RSS, Lauxen IAG. Burnout e fatores associados em docentes da Universidade Federal do Rio de Janeiro. Saúde em Redes. 2016; 2(1):97-116.

13. Massa LDB, Silva TSS, Sá ISVB, Barreto BCS, Almeida PHQT, Pontes TB. Síndrome de Burnout em professores universitários. Rev Ter Ocup Univ. 2016;27(2):180-9.

14. Faragher EB, Cass M, Cooper CL. The relationship between job satisfaction and health: a meta-analysis. Occup Environ Med. 2005;62(2):105-12.

15. Dalcin L, Carlotto MS. Síndrome de Burnout em professores no Brasil: considerações para uma agenda de pesquisa. Psicol. Rev. 2017; 23(2):745-71.

16. Brasil. Portaria de Consolidação $\mathrm{n}^{\circ}$ 4, de 28 de setembro de 2017. Anexo V - Capítulo I e Portaria de Consolidação $n^{0} 5$ - ANEXO XLIII) - Lista Nacional de Notificação Compulsória.

17. Fleck MPA, Louzada S, Xavier M, Chachamovic E, Vieira G, Santos V, et al. Aplicação da versão em português do instrumento abreviado de avaliação da qualidade de vida "WHOQOL-Bref". Rev Saúde Pública. 2000; 34(2):178-183.

18. Grunfeld E, Whelan TJ, Zitzelsberger L, Willan AR, Montesanto B, Evans WK. Cancer care workers in Ontario: prevalence of burnout, job stress and job satisfaction. CMAJ 2000;163: 166-9.

19. Carlotto MS, Câmara SG. Análise fatorial do Maslach Burnout Inventory (MBI) em uma amostra de professores de instituições particulares. Psicologia em Estudo, v. 9, n. 3, p. 499-505, 2004.

20. Sanchez HM, Sanchez EGM, Barbosa MA, Guimarães EC, Porto CC. Impacto da saúde na qualidade de vida e trabalho de docentes universitários de diferentes áreas de conhecimento. Ciênc. saúde coletiva. 2019; 24(11): 4111-22.

21. Tolomeu R, Tavares FS, Monteiro IP, Camargos GL, Correa AAM. Qualidade de vida e estresse em professores de uma instituição de ensino superior do interior de Minas Gerais. Rev Cientifica Fagoc Saúde. 2017;2: 9-15.

22. Ministério da Saúde. Vigitel. Vigilância de fatores de risco e proteção para doenças crônicas por inquérito telefônico. Brasília. Ministério da Saúde. 2017. 130p.

23. Hirshkowitz M, Whiton K, Albert SM, Alessi C, Bruni O, DonCarlos L, et al. National Sleep Foundation's sleep time duration recommendations: methodology and results summary. Sleep Health 2015;1(1): 40-43.

24. Martins PJF, Mello MT, Tufik S. Exercício e sono. Rev Bras Med Esporte. 2001;7(1):2836 .

25. Koetz LCE. Qualidade de vida de professores de instituições de ensino superior comunitárias: relações entre ambiente e saúde [Dissertação]. Lageado: Centro Universitário UNIVATES; 2011.

26. Ibeu: índice de bem-estar urbano / organização Luiz Cesar de Queiroz Ribeiro, Marcelo Gomes Ribeiro. - 1. ed. - Rio de Janeiro: Letra Capital, 2013.

27. Ferreira MDL, Medeiros JCS, Nogueira ER, Assis EV, Dias MJ. Síndrome de Burnout em docentes do ensino superior. Rev. e-ciência, 2020; 8(2): 10-17.

28. Maslach C. Maslach Burnout Inventory. 2 ed. Palo Alto: CPP; 1986.

29. Bedoya, EA, Veja NE, Severiche CA, Meza MJ. Síndrome de Quemado (Burnout) en Docentes Universitarios: El Caso de un Centro de Estudios del Caribe Colombiano. Formación universitaria, 2017;10(6): 51-58.

30. Leite TIA, Fernandes JPC, Araújo FLC, Pereira XBF, Azevedo DM, Lucena EES. Prevalência e fatores associados da síndrome de Burnout em docentes universitários. Rev Bras Med Trab. 2019;17(2):170-9. 
31. Carlotto MS, Palazzo LS. Síndrome de Burnout e fatores associados: um estudo epidemiológico com professores. Cad. Saúde Pública. 2006; 22:1017-26.

32. Servilha EAM. Estresse em professores universitários na área de fonoaudiologia. Rev Ciênc Méd. 2005;14(1):43-52.

33. Cardoso CGLV, Costa NMSC. Factors connected with professional satisfaction and dissatisfaction among nutrition teacher. Cien Saude Colet. 2016; 21(8): 2351-2364.

34. Bertaci AC, Santos BB, Coelho AT, Suda EY. Síndrome de Burnout e nível geral de saúde em professores universitários. Neurobiologia. 2011;74(1): 167-187.

35. Silva FM, Silva N, Martini CM. Síndrome de Burnout em professores da Escola Polo José de Anchieta e da Escola Estadual Cora Coralina na cidade de Ariquemes. Rev Fiar. 2013; 2(1):187-202.

\section{Como citar este artigo:}

Miranda IMM, Tavares HHF, Silva HRS, Braga MS, Santos RO, Silva RCD, Guerra HS. Avaliação da qualidade de vida e síndrome de burnout em professores universitários. Rev. Aten. Saúde. 2021; 19(69): 83-92. 